\title{
A Numerical Approach to Angular Distributions in Hadronic Cascades
}

\author{
Tetiana Kozynets, $^{a, *}$ Anatoli Fedynitch ${ }^{b}$ and D. Jason Koskinen ${ }^{a}$ \\ ${ }^{a}$ Niels Bohr Institute, University of Copenhagen, \\ Blegdamsvej 17, 2100 Copenhagen Ø, Denmark \\ ${ }^{b}$ Institute for Cosmic Ray Research, University of Tokyo, \\ 5-1-5 Kashiwa-no-ha, Kashiwa, Chiba 277-8582, Japan \\ E-mail: tetiana.kozynets@nbi.ku.dk, afedyni@icrr.u-tokyo.ac.jp, \\ koskinen@nbi.ku.dk
}

Hadronic interactions of highly energetic projectiles in matter induce rich cascades of daughter particles, an example being atmospheric neutrinos produced in cosmic ray air showers. Fully analytical modelling of such cascades, due to the amount and the complexity of the coupled processes involved, is infeasible, while Monte Carlo simulations remain computationally expensive. These complications are mitigated in the numerical Matrix Cascade Equations (MCEQ) code, which reaches Monte Carlo-like precision at extremely low computational costs. Previously, the MCEQ framework has included longitudinal-only development of the hadronic cascades.

To accurately model secondaries at $\mathrm{MeV}-\mathrm{GeV}$ energies in particle cascades, we extend the onedimensional cascade equation solver to $2 \mathrm{D}$ by including angular development. The distributions are computed via the spectral methods and compared to those produced with the Monte Carlo cascade codes. The potential applications of this study include fast numerical calculations of particle fluxes in air showers and atmospheric lepton flux calculations, which will benefit simulation chains of the cosmic ray and neutrino experiments.

$37^{\text {th }}$ International Cosmic Ray Conference (ICRC 2021)

July 12th - 23rd, 2021

Online - Berlin, Germany

\footnotetext{
*Presenter
} 


\section{Introduction}

Hadronic interactions of energetic projectiles in matter produce a wealth of daughter particles, whose subsequent reinteractions and/or decays result in a hadronic cascade. Such processes are central to the evolution of the cosmic ray air showers and form a natural environment to probe fundamental particle physics across a wide range of energies, from $\mathrm{MeV}$ to $\mathrm{PeV}$. A particularly important byproduct of the atmospheric hadronic cascades are neutrinos, which, at the energies of $O(10 \mathrm{GeV})$ and below, provide the leading signal for neutrino oscillation measurements. The angular distributions of neutrinos at these energies depend on the complete three-dimensional treatment of atmospheric neutrino production [1-5]. This presents one of the many physics cases where including both longitudinal and lateral components into hadronic cascade modelling is necessary.

The modern hadronic cascade codes are predominantly Monte Carlo based. These include e.g. FLUKA [6], GEANT4 [7], and PHITS [8] general purpose simulation packages for arbitrary materials, as well as dedicated atmospheric air shower codes such as CORSIKA [9] and AIRES [10]. A sophisticated treatment of the individual particle interactions in such codes comes with high computational costs. The complexity of the Monte Carlo solvers also makes immediate and comprehensive benchmarking of the different codes against each other rather difficult. These limitations make the existence of a precise, fast, and customizable hadronic cascade code particularly appealing.

A natural mathematical formulation of the cascade development problem is through the coupled differential equations for particle propagation, interaction, and decay. A current state-of-the-art software employing such an approach is the Matrix Cascade Equations (MCEQ) code ${ }^{1}$, which formulates the transport equations in the matrix form [11-13]. Up until now, MCEQ has provided only longitudinal cascade development. To extend its applications to the low-energy atmospheric neutrino flux modelling as well as air-shower and radiation dose calculations, we focus on including the angular component into the MCEQ framework. This study demonstrates that multi-dimensional hadronic cascade development can be well modeled as a sequence of angular convolutions.

\section{Overview of the Matrix Cascade Equations and the MCEq Code}

The longitudinal evolution of a hadronic cascade is governed by the 1D multi-species Boltzmann transport equation. Discretizing this equation in energy allows one to put the probabilities of interaction and decay processes in a matrix form and to solve for the particle fluxes $\boldsymbol{\Phi}$ on a fixed energy grid. Explicitly, for a cascade particle $h$, the differential spectrum $\Phi_{E_{i}}^{h} \equiv \frac{\mathrm{d} N_{E_{i}}^{h}}{\mathrm{~d} E}$ evolves as a function of the traversed slant depth $X$ according to

$$
\begin{aligned}
\frac{\mathrm{d} \Phi_{E_{i}}^{h}(X)}{\mathrm{d} X}= & -\frac{\Phi_{E_{i}}^{h}(X)}{\lambda_{\mathrm{int}, E_{i}}^{h}}-\frac{\Phi_{E_{i}}^{h}(X)}{\lambda_{\mathrm{dec}, E_{i}}^{h}(X)} \\
& +\sum_{E_{k} \geq E_{i}} \sum_{l} \frac{c_{l\left(E_{k}\right) \rightarrow h\left(E_{i}\right)}}{\lambda_{\mathrm{int}, E_{k}}^{l}} \Phi_{E_{k}}^{l}(X)+\sum_{E_{k} \geq E_{i}} \sum_{l} \frac{d_{l\left(E_{k}\right) \rightarrow h\left(E_{i}\right)}}{\lambda_{\mathrm{dec}, E_{k}}^{l}(X)} \Phi_{E_{k}}^{l}(X) .
\end{aligned}
$$

The particle $h$ in the energy bin $E_{i}$ can undergo inelastic collisions in the target medium and decay into other species following (1a), with the corresponding probabilities defined by the interaction

\footnotetext{
${ }^{1}$ https://github.com/afedynitch/MCEq
} 
length $\lambda_{\text {int, } E_{i}}^{h}$ and the decay length $\lambda_{\mathrm{dec}, E_{i}}^{h}$. The same particle can also be produced by other cascade species $l$ with energies $E_{k} \geq E_{i}$ through interactions or decays as per (1b). The probabilities of producing the secondary $h$ are represented as the yield coefficients $c_{l\left(E_{k}\right) \rightarrow h\left(E_{i}\right)}$ and $d_{l\left(E_{k}\right) \rightarrow h\left(E_{i}\right)}$. The equations for the different particle species are coupled and can be solved in a matrix form by the forward propagation of Eq. (1) in $X$, which is further detailed in [11, 13].

In MCEQ, the coefficients $c_{l\left(E_{k}\right) \rightarrow h\left(E_{i}\right)}$ and $d_{l\left(E_{k}\right) \rightarrow h\left(E_{i}\right)}$ are derived directly from event generators by histogramming the yields of $h$ from an $l$-target collision or a decay of $l$ as a function of $E_{i} / E_{k}$. For $l$-target collisions, interaction models such as EPOS-LHC [14], URQMD [15], and DPMJET-III [16] can be employed, while PYTHIA 8 [17] is used to handle the kinematics of decays.

\section{Angular Cascade Development in the MCEq Framework: "2D MCEq"}

\subsection{Evolving the Cascades via Sequential Angular Convolutions}

In Section 2, the spectra $\Phi_{E_{i}}^{h}$ entering the cascade equation are integrated over the angle $\theta$ that the secondaries make with the primary axis. We now wish to evolve the spectra as a function of $\theta$ in addition to the slant depth $X$. In what follows, we will consider only forward-going particles, i.e. those with $0 \leq \theta \leq \pi / 2$, and assume azimuthal symmetry $w r t$. the initial particle direction. Defining $\phi_{E_{i}}^{h}(X, \theta) \equiv \frac{\mathrm{d} N_{E_{i}}^{h}(X, \theta)}{\mathrm{d} E \mathrm{~d} \theta}$, we can expand Eq. (1) as

$$
\begin{aligned}
\frac{\mathrm{d} \phi_{E_{i}}^{h}(X, \theta)}{\mathrm{d} X}= & -\frac{\phi_{E_{i}}^{h}(X, \theta)}{\lambda_{\mathrm{int}, E_{i}}^{h}}-\frac{\phi_{E_{i}}^{h}(X, \theta)}{\lambda_{\mathrm{dec}, E_{i}}^{h}(X)} \\
& +\sum_{E_{k} \geq E_{i}} \sum_{l} \int_{0}^{\pi / 2} \frac{\varsigma_{l\left(E_{k}, \theta^{\prime}\right) \rightarrow h\left(E_{i}, \theta\right)}}{\lambda_{\mathrm{int}, E_{k}}^{l}} \phi_{E_{k}}^{l}\left(X, \theta^{\prime}\right) \mathrm{d} \theta^{\prime} \\
& +\sum_{E_{k} \geq E_{i}} \sum_{l} \int_{0}^{\pi / 2} \frac{\delta_{l\left(E_{k}, \theta^{\prime}\right) \rightarrow h\left(E_{i}, \theta\right)}}{\lambda_{\mathrm{dec}, E_{k}}^{l}(X)} \phi_{E_{k}}^{l}\left(X, \theta^{\prime}\right) \mathrm{d} \theta^{\prime} .
\end{aligned}
$$

The new double-differential yield coefficients are normalized through integration over $\theta$ to match the particle yield coefficients of $1 \mathrm{D}$ MCEQ

$$
c_{l\left(E_{k}\right) \rightarrow h\left(E_{i}\right)} \equiv \int_{0}^{\pi / 2} S_{l\left(E_{k}, \theta^{\prime}\right) \rightarrow h\left(E_{i}, \theta\right)} \mathrm{d}\left(\theta-\theta^{\prime}\right)=\int_{0}^{\pi / 2} \varsigma_{l\left(E_{k}, 0\right) \rightarrow h\left(E_{i}, \theta\right)} \mathrm{d} \theta .
$$

As we restrict ourselves to $0 \leq \theta \leq \pi / 2$ and set particle fluxes outside this domain to 0 , the integration bounds may well be extended to the periodic $-\pi . . \pi$ interval. This lets us formulate the collision integral in terms of the circular convolution operator $\circledast$ :

$$
\int_{0}^{\pi / 2} \varsigma_{l\left(E_{k}, \theta^{\prime}\right) \rightarrow h\left(E_{i}, \theta\right)} \phi_{E_{k}}^{l}\left(X, \theta^{\prime}\right) \mathrm{d} \theta^{\prime} \equiv\left[\varsigma_{l\left(E_{k}\right) \rightarrow h\left(E_{i}\right)} \circledast \phi_{E_{k}}^{l}\right](\theta)
$$

The inclusion of the angular variable then becomes a straightforward extension of the $1 \mathrm{D}$ MCEQ functionality. As before, one proceeds by simulating the $l\left(E_{k}\right)$-target collision events or the decays of $l\left(E_{k}\right)$ in a Monte Carlo event generator. An extra step in the 2D case is to keep track of the angular distribution $\varsigma_{l\left(E_{k}\right) \rightarrow h\left(E_{i}\right)}(\theta)$ when histogramming the yields of the particle $h$ in the $l\left(E_{k}\right) \rightarrow h\left(E_{i}\right)$ 


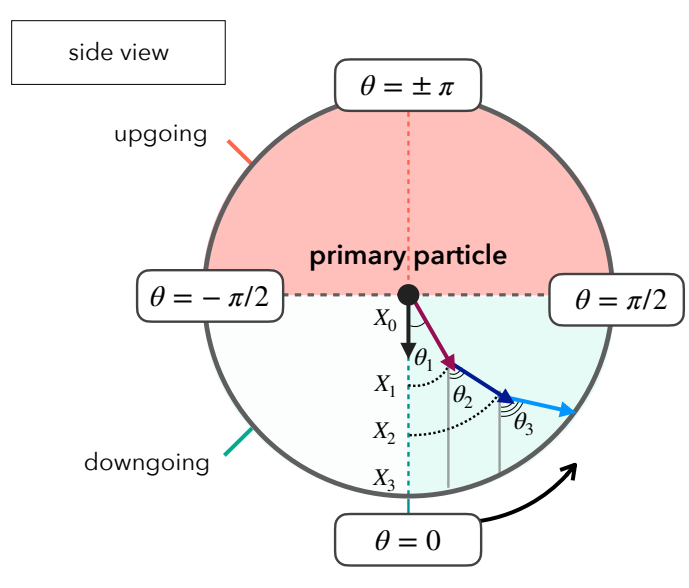

circular convolution $\Longrightarrow$ Fourier transform top-down view $\otimes$ towards ground

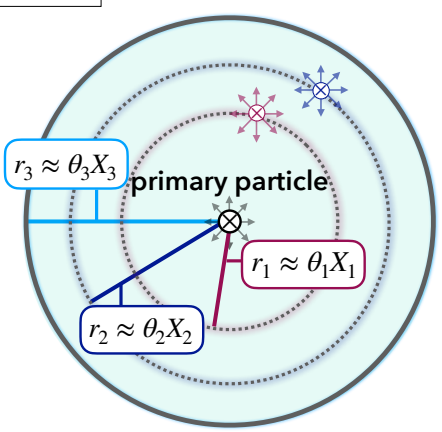

radial convolution $\Longrightarrow$ Hankel transform

Figure 1: Two different views on the evolution of a hadronic cascade in the longitudinal $(X)$ and angular $(\theta)$ dimensions. In both cases, the primary particle (e.g. a cosmic ray proton) is injected at $\theta=0$ and $X_{0}=0$. At a depth $X_{i}$, the secondaries make an angle $\theta_{i}$ with the primary axis. On the left panel, $\theta$ is treated as a quasi-periodic circular variable, which we restrict to the forward $(|\theta| \leq \pi / 2)$ region. On the right panel, $\theta_{i}$ is interpreted as the radius of the circle containing the secondaries at $X_{i}$. This leads to the appearance of either circular or radial convolution operators in Eq. (4), which correspondingly translate to either Fourier or Hankel transforms in the spectral formulation of the problem (see Section 3.2).

process. The probability density of the outgoing secondary angle with respect to the primary direction then acts as a convolution kernel for Eq. (4).

Note that if we instead defined $\phi_{E_{i}}^{h}(X, \theta) \equiv \frac{\mathrm{d} N_{E_{i}}^{h}(X, \theta)}{\mathrm{d} E \sin \theta \mathrm{d} \theta} \approx \frac{\mathrm{d} N_{E_{i}}^{h}(X, \theta)}{\mathrm{d} E \theta \mathrm{d} \theta}$ for small $\theta$, the linear integration in $\theta$ in Eqs. (2) to (4) would be replaced by the radial one: $\mathrm{d} \theta \rightarrow \theta \mathrm{d} \theta$, and the " $1 \mathrm{D}$ " circular convolution operator $(\circledast)$ would change to the " $2 \mathrm{D}$ " radial convolution operator $(* *)$. These two different approaches to the angular evolution of particle cascades are illustrated in Fig. 1.

\subsection{The Spectral Convolution Method}

Depending on the energies involved in a hadronic cascade, the angle $\theta$ that a daughter particle makes with its immediate parent may vary significantly and span several orders of magnitude. Thus, if the convolutions were performed on a uniform $\theta$ grid, the latter would have to be discretized very finely to capture the processes at all angular scales, presenting a major computational challenge. A convenient way to get around this complication is to bring the $2 \mathrm{D}$ cascade equation to the spectral domain, where the particle fluxes are given as a function of frequency $f$. Specifically, in case of the circular convolutions, one can Fourier-transform both sides of Eq. (2), so that $\tilde{\phi}_{E_{i}}^{h}(X, f) \equiv \mathcal{F}\left[\phi_{E_{i}}^{h}(X, \theta)\right](f)$ and $\tilde{\varsigma}_{l\left(E_{k}\right) \rightarrow h\left(E_{i}\right)}(f) \equiv \mathcal{F}\left[\varsigma_{l\left(E_{k}\right) \rightarrow h\left(E_{i}\right)}(\theta)\right](f)$. Then, via the convolution theorem, the circular convolution $\circledast$ transforms into simple multiplication: 


$$
\begin{aligned}
\frac{\mathrm{d} \tilde{\phi}_{E_{i}}^{h}(X, f)}{\mathrm{d} X}= & -\frac{\tilde{\phi}_{E_{i}}^{h}(X, f)}{\lambda_{\mathrm{int}, E_{i}}^{h}-\frac{\tilde{\phi}_{E_{i}}^{h}(X, f)}{\lambda_{\mathrm{dec}, E_{i}}^{h}(X)}} \\
& +\sum_{E_{k} \geq E_{i}} \sum_{l} \frac{\left[\tilde{s}_{l}\left(E_{k}\right) \rightarrow h\left(E_{i}\right) \cdot \tilde{\phi}_{E_{k}}^{l}\right](f)}{\lambda_{\mathrm{int}, E_{k}}^{l}}+\sum_{E_{k} \geq E_{i}} \sum_{l} \frac{\left[\tilde{\delta}_{l\left(E_{k}\right) \rightarrow h\left(E_{i}\right)} \cdot \tilde{\phi}_{E_{k}}^{l}\right](f)}{\lambda_{\mathrm{dec}, E_{k}}^{l}(X)} .
\end{aligned}
$$

For the 2D radial convolutions (as in the right panel of Fig. 1), the equivalent transform is the Hankel transform $\mathcal{H}[18,19]$. With discrete transforms, a finite number $N$ of frequencies $f_{n}$ is implied in Eq. (5), and the multiplication $\left[\tilde{\varsigma}_{l\left(E_{k}\right) \rightarrow h\left(E_{i}\right)} \cdot \tilde{\phi}_{E_{k}}^{l}\right](f)$ is performed independently for each $f_{n}$. This means that 2D cascade equation in MCEQ will preserve the matrix form and the computational advantages of the 1D MCEQ solution, albeit now requiring one to solve $N$ matrix equations in parallel for each frequency mode or assembling a larger block-diagonal sparse matrix.

\section{Validation and Benchmarking}

To validate the numerical approach developed in Section 3.2, we solve Eq. (5) for a $100 \mathrm{GeV}$ proton primary injected into the Earth's atmosphere at $\theta_{0}=0$ and the altitude $h_{0}=112.5 \mathrm{~km}$ $\left(X_{0}=0 \mathrm{~g} \mathrm{~cm}^{-2}\right)$. For the atmospheric profile, we choose the Linsley parametrization of the US Standard atmosphere. The combination of DPMJET-III $19.1^{2}\left(E_{i} \leq 80 \mathrm{GeV}\right)$ and EPOS-LHC $\left(E_{i}>80 \mathrm{GeV}\right)$ hadronic interaction models is used to generate the yields of the secondary particles as a function of their energy and angle relative to their immediate primary. To run the interaction models we use the new IMPY interface ${ }^{3}$. The energy grid is log-spaced following [11] and extends from $1 \mathrm{GeV}$ to $2 \mathrm{TeV}$. The electromagnetic processes, including multiple scattering of the shower muons, as well as the deflection of charged particles in the geomagnetic field are not taken into account. For both of the convolution approaches, we use $N \simeq 400$ frequency modes $f_{n}$, and obtain the final fluxes in the $\theta$ space through the respective inverse transforms $\left(\mathcal{F}^{-1}\right.$ or $\left.\mathcal{H}^{-1}\right)$ of $\tilde{\phi}_{E_{i}}^{h}\left(X, f_{n}\right)$.

For benchmarking, we use the CORsiKA Monte Carlo code, v.7.7410 [9], and perform 170,000 simulations of proton showers using the same atmospheric profile. As in MCEQ, EPOS-LHC is the high-energy ( $\geq 80 \mathrm{GeV}$ ) hadronic interaction model. At lower energies, CORSIKA is set to URQMD since DPMJET-III is not supported. The geomagnetic field is disabled, while the muon multiple scattering remains present in the simulations.

Fig. 2 shows the resulting angular distributions of the secondary muons from CORSIKA and those obtained via the method from Section 3.2 ("2D MCEQ"). We find a good agreement between the angular distributions obtained with 2D MCEQ (via the radial convolution and the Hankel transform) and CORSIKA for all altitudes and energy bins considered. These results are further cross-checked against the AIRES Monte Carlo [10] and the circular (Fourier) convolution method for 2D MCEQ in Fig. 3. The angle-integrated muon spectra are given in Fig. 4. We observe that the angular distributions returned by the circular convolution method are in a worse agreement with both CORSIKA and AIRES than the radial convolution. With the circular convolution, one consistently obtains more widely spread secondaries than predicted by both Monte Carlo codes. This is an intuitive

${ }^{2}$ https://github.com/DPMJET

3https://github.com/impy-project 
2D MCEq (radial) vs. CORSIKA

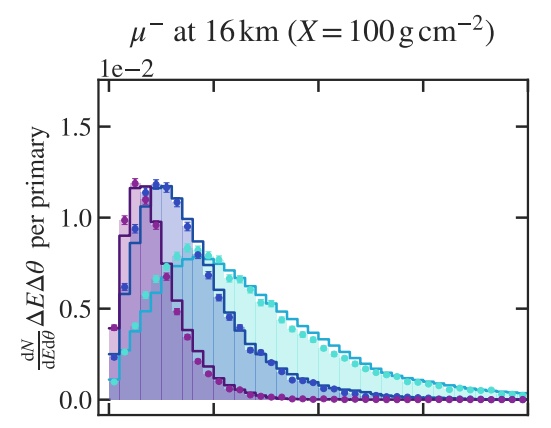

$\mu^{-}$at $6 \mathrm{~km}\left(X=500 \mathrm{~g} \mathrm{~cm}^{-2}\right)$

$\mu^{-}$at $0 \mathrm{~km}\left(X=1000 \mathrm{~g} \mathrm{~cm}^{-2}\right)$
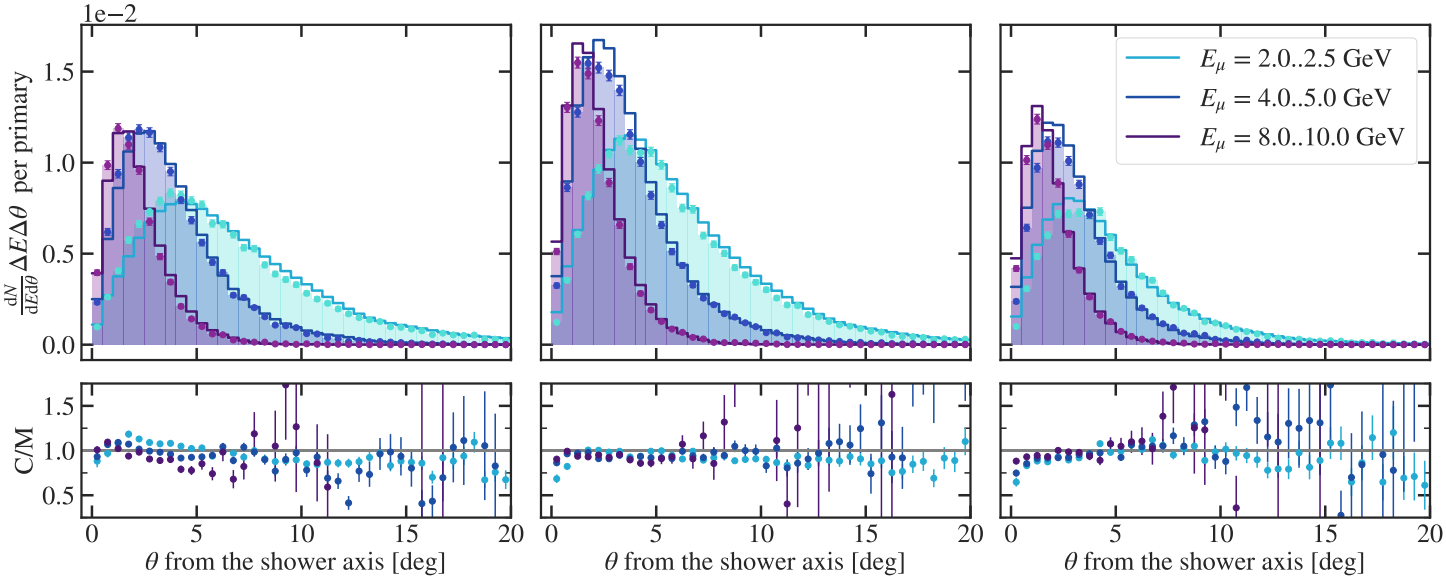

Figure 2: The angular distributions of secondary muons at three different altitudes: the numerical solution of Eq. (5) via MCEQ (solid line) as compared to the output of the CORSIKA simulations (shaded histogram). The primary particle inducing the hadronic cascade is a $100 \mathrm{GeV}$ proton. The coRsIKA results are shown with their respective statistical errorbars. The MCEQ solution uses the radial (Hankel) convolution approach. The bottom panel provides the ratio of the CORSIKA:MCEQ angular spectra integrated within the $0.5^{\circ}$ bins

consequence of the fact that the secondary particle angle can only increase at each propagation step (i.e. the rotation illustrated in the left panel of Fig. 1 is performed strictly counter-clockwise). At $\theta=0$, such a deficiency of the circular convolution approach turns out to be particularly problematic, and the bias of the respective angular spectra is most evident in the low-energy hadrons (top left panel of Fig. 3). This is expected as the angles of the hadronic interaction secondaries relative to their primaries are larger than the angles of the decay daughters relative to their parents. As the result, each circular convolution introduces a larger error for interactions than for decays.

Finally, we note that the CORSIKA and AIRES distributions are not fully in agreement with each other. This discrepancy could be attributed to the fact that AIRES employs a different hadronic interaction model, namely the Hillas splitting algorithm [20], at low energies, while the high-energy hadronic interaction model (EPOS-LHC) is matched with those of CORSIKA and MCEQ. At present, elucidating the origin of any disagreement between the two Monte Carlo codes falls beyond the scope of this work.

\section{Conclusions}

This study focused on extending MCEQ, a state-of-the-art numerical code for hadronic cascade evolution, to two dimensions. By treating angular development as a sequence of convolutions, we naturally incorporated it into the MCEQ framework. We compared two spectral convolution methods and benchmarked them against two Monte Carlo cascade codes, using an example of a protoninduced air shower. A very good agreement between the "2D MCEQ" solution and the output from a standard Monte Carlo code was reached. This suggests that our tool has a significant potential to be used as a fast and accurate alternative to the Monte Carlo cascade development approaches. 


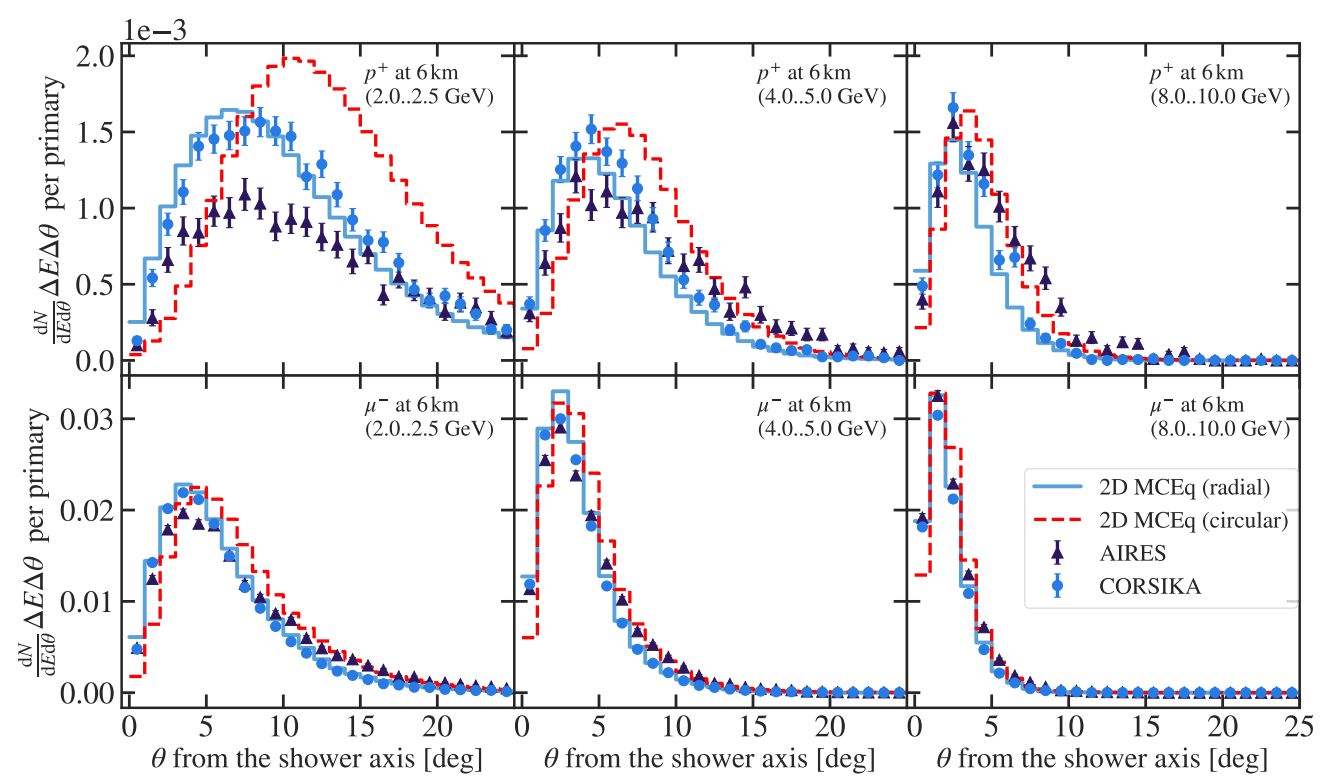

Figure 3: Comparison of the angular distributions of the secondary particles in a $100 \mathrm{GeV}$ proton air shower as evaluated by the two numerical methods from this study (solid and dashed lines) and the two benchmark Monte Carlo codes (barred markers). The best agreement is reached between the 2D MCEQ solution with the radial convolution method and CORSIKA, which is most prominent in low-energy muons and protons.

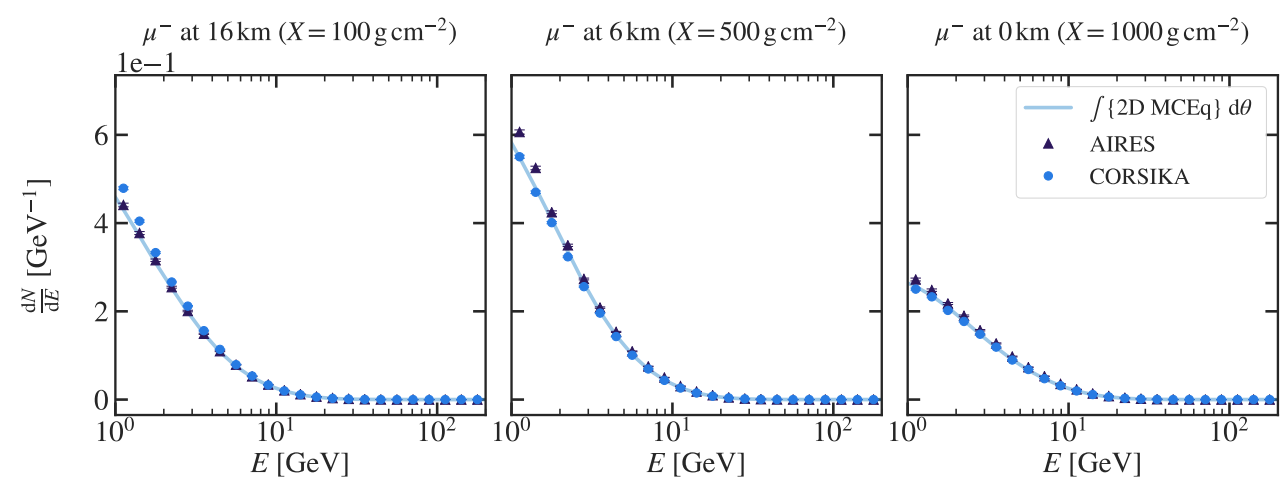

Figure 4: Angle-integrated muon spectra from 2D MCEQ (radial convolution method) as compared to those from CORSIKA and AIRES Monte Carlo simulations. All three codes are found to be in agreement.

\section{Acknowledgements}

TK and DJK acknowledge support from the Carlsberg Foundation (project no. 117238). AF acknowledges support from the JSPS (KAKENHI 19F19750).

\section{References}

[1] V. Agrawal, T.K. Gaisser, P. Lipari and T. Stanev, Phys. Rev. D 53 (1996) 1314.

[2] G.D. Barr, T.K. Gaisser, P. Lipari, S. Robbins and T. Stanev, Phys. Rev. D 70 (2004) 023006. 
[3] M. Honda, T. Kajita, K. Kasahara, S. Midorikawa and T. Sanuki, Phys. Rev. D 75 (2007) 043006.

[4] M. Honda, M.S. Athar, T. Kajita, K. Kasahara and S. Midorikawa, Phys. Rev. D 92 (2015) 023004.

[5] P. Lipari, Astropart. Phys. 14 (2000) 153.

[6] G. Battistoni, J. Bauer, T.T. Boehlen, F. Cerutti, M.P.W. Chin, R. Dos Santos Augusto et al., Front. Oncol. 6 (2016) .

[7] S. Agostinelli et al., Nucl. Instrum. Methods Phys. Res. A 506 (2003) 250.

[8] T. Sato et al., J. Nucl. Sci. Technol. 55 (2018) 684.

[9] D. Heck, J. Knapp, J.N. Capdevielle, G. Schatz and T. Thouw, Tech. Rep. FZKA-6019 (1998).

[10] S.J. Sciutto, arXiv:astro-ph/9911331 (2019) .

[11] A. Fedynitch, R. Engel, T.K. Gaisser, F. Riehn and T. Stanev, arXiv:1503.00544 (2015) .

[12] A. Fedynitch, Cascade equations and hadronic interactions at very high energies, Ph.D. thesis, KIT, Karlsruhe, Dept. Phys., Nov., 2015.

[13] A. Fedynitch, F. Riehn, R. Engel, T.K. Gaisser and T. Stanev, Phys. Rev. D 100 (2019) 103018.

[14] T. Pierog, I. Karpenko, J.M. Katzy, E. Yatsenko and K. Werner, Phys. Rev. C 92 (2015) 034906.

[15] M. Bleicher, E. Zabrodin, C. Spieles, S.A. Bass, C. Ernst, S. Soff et al., J. Phys. G: Nucl. Part. Phys. 25 (1999) 1859.

[16] S. Roesler, R. Engel and J. Ranft, in Advanced Monte Carlo for Radiation Physics, Particle Transport Simulation and Applications, A. Kling, F.J.C. Baräo, M. Nakagawa, L. Távora and P. Vaz, eds., (Berlin, Heidelberg), pp. 1033-1038, Springer, 2001.

[17] T. Sjöstrand, S. Ask, J.R. Christiansen, R. Corke, N. Desai, P. Ilten et al., Comput. Phys. Commun. 191 (2015) 159.

[18] N. Baddour, J. Opt. Soc. Am. A 26 (2009) 1767.

[19] M. Guizar-Sicairos and J.C. Gutiérrez-Vega, JOSA A 21 (2004) 53.

[20] A.M. Hillas in Proc. of the Paris Workshop on Cascade Simulations, J. Linsley and A.M. Hillas, eds., p. 39 (1981). 